Ciencias agrarias/Agricultural Sciences

Cienc Tecn UTEQ (2019) 12(1) p 19-30 ISSN 1390-4051; e-ISSN 1390-4043 (c) (i) ()()

doi:https://doi.org/10.18779/cyt.v12i1.316

\title{
Evaluación agronómica de genotipos de quinua (Chenopodium quinoa Willd.) en condiciones agroclimáticas en la zona de Mocache
}

\author{
Agronomic evaluation of quinoa genotypes (Chenopodium quinoa Willd.) \\ in agroclimatic conditions in the Mocache zone
}

Camilo Mestanza Uquillas ${ }^{1}$, Katiuska Zambrano Calderón ${ }^{1}$, John Pinargote Alava ${ }^{1}$, Diana Veliz Zamora ${ }^{1}$, Gregorio Vásconez Montufar ${ }^{1}$, Nieves Fernández-García ${ }^{2}$, Enrique Olmos².

${ }^{1}$ Facultad de Ciencias Pecuarias de la Universidad Técnica Estatal de Quevedo-(UTEQ), km 7 1/2 vía QuevedoEl Empalme. Mocache, Los Ríos, Ecuador.

${ }^{2}$ Department of Stress Biology and Plant Pathology, Centro de Edafología y Biología Aplicada del Segura (CEBAS-CSIC), Murcia, España.

Email: autor de correspondencia: cmestanza@uteq.edu.ec(0000-0001-9299-170X)

Rec.: 25.01.2019. Acept.: 20.06.2019.

Publicado el 30 de junio de 2019

\section{Resumen}

$\mathrm{L}^{2}$ a quinua (Chenopodium quinoa Willd.) se ha cultivado principalmente en la cordillera de los Andes en Bolivia, Perú, Ecuador y Colombia, fue uno de los principales alimentos de los pueblos andinos preincaicos e incaicos. Las bondades de la quinua radican en su alto valor nutricional por su contenido de proteína y su gran plasticidad fenotípica. La presente investigación se realizó en la Finca Experimental "La María” de la Universidad Técnica Estatal de Quevedo, ubicada en la provincia de Los Ríos. El objetivo del trabajo fue analizar las características agronómicas de los genotipos de quinua en condiciones ambientales de la costa central. Se aplicó un diseño completamente al azar con 21 tratamientos (genotipos) y tres repeticiones. Las variables agronómicas evaluadas mostraron diferencias estadísticas significativas $(\mathrm{p}<0.05)$. En la variable altura de planta destacó el genotipo 48 con $154.17 \mathrm{~cm}$ a los 90 días. Por otra parte, en la determinación de días a la cosecha el genotipo más tardío fue el genotipo RGG con 143 días, mientras el genotipo más precoz fue el 42 con 90 días a la cosecha. En la variable peso de 1,000 semillas los resultados alcanzados demostraron que el genotipo Faro 2 destacó ligeramente con un registro de $2.58 \mathrm{~g}$. Finalmente, en cuanto al rendimiento por planta (g) el mayor registro lo obtuvo el genotipo O-5 con 143.15 g planta $^{-1}$. Los datos registrados en las distintas variables demuestran viabilidad en la producción de quinua y debido a su amplia adaptabilidad, la convierten en una valiosa alternativa de diversificación para la costa ecuatoriana.

Palabras claves: costa, genotipos, morfología, adaptación, rendimiento.

\section{Abstract}

( uinoa (Chenopodium quinoa Willd.) has been cultivated mainly in the Andean mountain range in Bolivia, Peru, Ecuador and Colombia, it was one of the main foods of the pre-Inca and Inca peoples. The benefits of quinoa lie in its high nutritional value with a protein content and its great phenotypic plasticity. The present investigation was carried out in the Experimental Farm "La María" of the State Technical University of Quevedo, located in the Province of Los Ríos. The objective of the work was to analyze the agronomic characteristics of quinoa genotypes in environmental conditions of the central coast. A completely randomized design was applied with 21 treatments (genotypes) and three repetitions. The agronomic variables evaluated showed significant statistical differences $(p<0.05)$. In the height variable of the plant it highlighted genotype 48 with $154.17 \mathrm{~cm}$ to 90 days. On the other hand, in the determination of days to harvest the latest genotype was RGG genotype with 143 days, while the earliest genotype was 42 with 90 days to harvest. Variable weight of 1,000 seeds the results showed that the genotype Faro 2 pointed out slightly with a record of $2.58 \mathrm{~g}$. Finally, in terms of performance per plant $(\mathrm{g})$ the largest recorded genotype obtained it O-5 with $143.15 \mathrm{~g} \mathrm{plant}^{-1}$. The data recorded in the different variables demonstrate viability in the production of quinoa and due to its wide adaptability, make it a valuable diversification alternative for the coastal region.

Key words: coast, genotypes, morphology, adaptation, performance. 


\section{Introducción}

La quinua (Chenopodium quinoa Willd.) es una planta autóctona de los Andes, su centro de origen se encuentra en algún valle de la zona andina y la mayor variabilidad se observa a orillas del lago Titicaca. En su historia se reconoce que fue utilizada como alimento desde hace 5,000 años (Fuentes et al., 2009; Zurita-Silva et al., 2014), su importancia reside en la alta calidad como alimento, la utilización completa de la planta y su amplia adaptación a condiciones agroecológicas. También está considerada como el alimento más completo para la nutrición humana basada en proteínas de la mejor calidad en el reino vegetal por el balance ideal de sus aminoácidos esenciales (Mujica y Jacobsen, 2006; Lutz et al., 2013).

El alto potencial agrícola y nutritivo ha causado el interés por la quinua, incrementándose la superficie de cultivo en los últimos años hasta convertirla en alternativa de diversificación para la región andina (Rojas et al., 2010) y Sudamérica en general (Belmonte et al., 2018). En la actualidad es considerada un producto "estrella" en el mundo por sus propiedades nutritivas y medicinales. Presenta diferentes variedades, y es el único entre los cereales que posee todos los aminoácidos, además de ser la única alternativa entre los alimentos de origen vegetal para reemplazar la proteína animal (Thanapornpoonpong et al., 2008; Hernández, 2015) although it is also used for animal feeding. It has high nutritional value and offers high biological content proteins and low glycemic index carbohydrates, which makes it adequate for patients with diabetes mellitus.

$\mathrm{La}$ quinua en Ecuador fue un alimento muy apreciado por poblaciones aborígenes. Los Cañarís cultivaban la planta antes de la llegada de los españoles, a fines del siglo XVI, ha persistido entre los campesinos del área de Carchi, Imbabura, Pichincha, Cotopaxi, Chimborazo, Loja, Azuay, entre otras localidades de la región andina ecuatoriana. Por la ubicación geográfica del Ecuador y sus características climáticas, la quinua no es afectada por plagas o enfermedades importantes (Rojas et al., 2010).

Recientemente, se ha revalorizado aún más en el mercado nacional e internacional motivado por las iniciativas relacionadas a la promoción en el Año Internacional de la Quinua en el 2013, lo que ha conllevado a un incremento extraordinario en el área sembrada en los principales países de producción como son: Perú, Bolivia y Ecuador. Por ejemplo, en Perú, el área sembrada creció de 25,601 ha en el año 2001 a 68,037 ha en el 2014. Así mismo, su producción tradicionalmente en los Andes, se ha expandido a nuevas áreas de producción, siendo actualmente producida en regiones costeras. En Bolivia, la superficie cultivada pasó de 37,223 ha en el 2001 a 173,960 ha en el año 2014. Mientras que, en Ecuador, en el año 2001 se sembraban solo 650 ha de este cultivo, para el 2014 la superficie sembrada se incrementó a 1,230 ha (Cruces, 2016). El incremento de la superficie producida en quinua en los países andinos, ha sido principalmente para satisfacer la demanda mundial, es decir, con fines de exportación, y una mínima parte para consumo interno.

El informe de "Rendimientos de quinua en el Ecuador 2016" del MAGAP, reflejó el nivel de productividad a nivel nacional, en el ciclo productivo (octubre 2015- agosto 2016). Los principales resultados obtenidos indican que la productividad de quinua a nivel nacional exhibe un rendimiento de $1.36 \mathrm{t} / \mathrm{h}$ (Alvarado y Martínez, 2015). Es así, que la base de datos estadísticos de la Organización de las Naciones Unidas para la Alimentación (FAOSTAT) indica que la producción de quinua en Ecuador durante el año 2015 fue de 12,707 toneladas, con una superficie cosechada de 7,148 ha, proyectándose a producir 16,000 ha de quinua (FAOSTAT, 2017).

Sin embargo, la demanda internacional de quinua sugiere mejorar la productividad e incrementar la superficie destinada su cultivo, consecuentemente diversificar las alternativas de cultivo e incrementar los ingresos del sector agrícola como lo indica el Instituto de Investigaciones Agropecuarias de Chile (INIA, 2015). En vista que Ecuador es un país en vías de desarrollo requiere diversificar la producción y contribuir al cambio de la matriz productiva mejorando el nivel económico y social de los productores. Para lograr tales objetivos es necesaria la búsqueda de nuevos genotipos de quinua seleccionados por su adaptabilidad y productividad. Los genotipos de quinua con los que cuenta Ecuador son los nativos de la región andina y ciertas semillas mejoradas por el Instituto Nacional de Investigaciones Agropecuarias de Ecuador (INIAP). Sin embargo, estas no tienen gran difusión en la zona costera del país.

Teniendo en cuenta estos antecedentes se realizó la presente investigación con la finalidad de evaluar 21 nuevos genotipos de quinua procedentes de Chile y Argentina, los cuales se establecieron en la zona de Mocache, provincia de Los Ríos.

\section{Materiales y métodos}

La presente investigación se llevó a cabo en La Finca Experimental "La María" propiedad de la Universidad Técnica Estatal de Quevedo, localizada en el Km 7.5, de la vía Quevedo - El Empalme, cantón Mocache, provincia de Los Ríos, durante los meses de agosto a diciembre del 2017, en la época seca. La ubicación geográfica es de $1^{0} 6^{\prime} 28^{\prime \prime}$ de latitud sur y $70^{\circ}$ 27 ' 13 " de longitud oeste, a una altura de $72 \mathrm{msnm}$. 
Bajo las siguientes características climáticas y edáficas: temperatura promedio $26^{\circ} \mathrm{C}$, humedad relativa $87.71 \%$, heliofanía 915.56 horas luz/año, precipitación anual 2,274.29 mm, y una evaporación promedio anual de $89.46 \mathrm{~mm}$, zona ecológica bosque húmedo tropical (bh $-\mathrm{T})$.

\section{Diseño experimental}

Se implementó un diseño completamente al azar (DCA) conformado por veintiún tratamientos y tres repeticiones, para un total de sesenta y tres parcelas (Cuadro 1). La interpretación de los resultados se realizó mediante el análisis de varianza (ADEVA) y la prueba de Tukey al 5\%.

\section{Manejo del cultivo}

La siembra de los genotipos se realizó durante la época seca mediante la implementación de bandejas germinadoras, hasta su respectivo establecimiento a los 16 días en el terreno de estudio, considerando que las plantas tuvieran dos hojas verdaderas y verificando que el suelo tuviera una humedad favorable para el trasplante (siendo suficiente un 30\% de humedad en el suelo), a una distancia entre plantas de $0.25 \mathrm{~m}$ y entre hileras de $0.30 \mathrm{~m}$, con una densidad de 133,333 plantas/ha. En cuanto a labores culturales posterior al establecimiento del cultivo en campo, se suministró el agua a las parcelas de forma manual hasta alcanzar capacidad de campo, en periodos de dos días por semana. A su vez, se llevó a cabo el control de maleza entre los días 45 y 60 , así como también se procedió a realizar un aporque manual, para de esta manera evitar volcamiento. Por último, se efectuó la aplicación de un estimulante para el crecimiento radicular denominado "Mas Raíz" tanto en la etapa germinativa, como en la etapa de crecimiento. Se emplearon solamente 60 plantas por cada tratamiento, es decir 20 por cada parcela, dado la disponibilidad de semillas de los genotipos empleados (Cuadro 1).

\section{Variables en estudio}

\section{Tipo de panoja}

Se determinó si eran amarantiformes, glomeruladas o intermedias.

\section{Color de hojas}

Se realizó antes de que inicie la floración en los distintos genotipos.

\section{Color de la flor}

Característica que se reflejó a medida que fueron apareciendo las primeras flores.

\section{Color del grano}

Esta variable morfológica se determinó posterior al trillado de las panojas, para de esta manera apreciar el grano libre de impurezas.

\section{Altura de planta $(\mathrm{cm})$}

De cada unidad experimental se midió la altura de tres plantas seleccionadas al azar a los 30, 60 y 90 días, después de la siembra se midieron desde el nivel del suelo hasta el ápice final de la planta.

\section{Diámetro del tallo $(\mathrm{cm})$}

Se tomaron tres plantas al azar de cada unidad experimental, de cada una de ellas se registró el grosor del tallo; lo cual se realizó con la ayuda de un calibrador.

\section{Días a la cosecha}

Se contabilizaron los días transcurridos desde la siembra, este dato se lo tomó hasta que por lo menos el $50+1$ de plantas de la parcela presentaran características de madurez fisiológica.

\section{Peso de mil semillas (g)}

De cada genotipo cosechado se contabilizaron 1000 semillas escogidas al azar, luego se pesaron en una balanza digital analítica.

\section{Rendimiento por planta (g)}

Se seleccionaron las panojas de tres plantas al azar de cada unidad experimental, luego de realizar la trilla y limpieza se pesó toda la semilla obtenida, la medida se registró en gramos por planta ( $\mathrm{g}$ planta $\left.{ }^{-1}\right)$.

\section{Resultados y discusión}

\section{Tipo de panoja}

La inflorescencia o panoja que presentaron los genotipos en su mayoría son: glomeruladas las cuales obtuvieron un $62 \%$, amarantiforme con $29 \%$ y mixtas con 9\% respectivamente (Figura 1) (Cuadro 2). Cabe señalar que el tamaño y tipo de panojas es un componente del rendimiento en quinua y una variación amplia significativa en este rasgo puede implicar diferencias importantes en el rendimiento de grano (Long, 2016). Las de tipo amarantiforme, presentan desventaja frente a las de tipo glomerulado, ya que el rendimiento en grano es menor. Cortés y Rubiano (2007) describen en sus resultados sobre el tipo de panoja que un alto porcentaje de plantas son panojas de tipo amarantiforme, en genotipos sembrados en Cundinamarca, Colombia. 
Cuadro 1. Esquema del tratamiento.

\begin{tabular}{|c|c|c|c|c|c|}
\hline Tratamiento & Genotipo & Repeticiones & TUE/Plantas & $\begin{array}{l}\text { No Plantas/ } \\
\text { Tratamiento } \\
\end{array}$ & Origen \\
\hline 1 & 26 & 3 & 20 & 60 & Chile \\
\hline 2 & 36 & 3 & 20 & 60 & Chile \\
\hline 3 & 41 & 3 & 20 & 60 & Chile \\
\hline 4 & 42 & 3 & 20 & 60 & Chile \\
\hline 5 & 48 & 3 & 20 & 60 & Chile \\
\hline 6 & 49 & 3 & 20 & 60 & Chile \\
\hline 7 & 52 & 3 & 20 & 60 & Chile \\
\hline 8 & 54 & 3 & 20 & 60 & Chile \\
\hline 9 & $0-1$ & 3 & 20 & 60 & Chile \\
\hline 10 & $0-2$ & 3 & 20 & 60 & Chile \\
\hline 11 & $0-3$ & 3 & 20 & 60 & Chile \\
\hline 12 & $0-4$ & 3 & 20 & 60 & Chile \\
\hline 13 & $0-5$ & 3 & 20 & 60 & Chile \\
\hline 14 & $0-6$ & 3 & 20 & 60 & Chile \\
\hline 15 & $0-7$ & 3 & 20 & 60 & Chile \\
\hline 16 & $0-8$ & 3 & 20 & 60 & Chile \\
\hline 17 & $0-9$ & 3 & 20 & 60 & Chile \\
\hline 18 & $0-10$ & 3 & 20 & 60 & Chile \\
\hline 19 & RGG & 3 & 20 & 60 & Chile \\
\hline 20 & $\mathrm{~J} 4-010$ & 3 & 20 & 60 & Argentina \\
\hline 21 & Faro 2 & 3 & 20 & 60 & Chile \\
\hline Total & & & & 1260 & \\
\hline
\end{tabular}

TUE: Tamaño de la Unidad Experimental 


\section{Color de hojas}

Los colores que más predominan en las hojas de los genotipos evaluados son: amarillo verdoso en un $43 \%$, seguido de las hojas de coloración rosado con un $24 \%$, posteriormente las hojas de color amarilla alcanzan el $19 \%$ y por último las de coloración naranja que representan el 14\% (Figura 1) (Cuadro 2). El color de la hoja en los genotipos de quinua predominantemente es verde; en algunas variedades puede observarse hojas de color verde-púrpura. A la madurez las láminas se tornan amarillas, naranjas, rosadas, rojas o púrpuras (Gómez y Aguilar, 2016), aunque el color de la hoja es muy variable dependiendo del genotipo (Bazile, 2013). De acuerdo con Delgado, Palacios y Betancourt (2009), en el área de Nariño, Colombia existe un ecotipo predominante con hojas de color verde claro, de porte alto, grano muy blanco y dulce.

\section{Color de la flor}

Las coloraciones que interactuaron dentro de la variable color de la flor son: verde con un 57\%, seguida de la flor de coloración amarilla con un 19\%, posteriormente las flores de color rojo alcanzan el $14 \%$ y por ultimo las de coloración rosa-amarillo que representan el 10\% (Figura 1) (Cuadro 2).

\section{Color del grano}

La diversidad de colores de granos de quinua es representada de la siguiente manera: el color amarillo con un $43 \%$, blanco con un $28 \%$, rojo con un $24 \%$ y negro con un 5\% (Cuadro 3). Así mismo Marca et al., (2015), menciona que aquellas variedades y ecotipos de semilla de color blanco son más susceptibles al ataque de aves plaga (pájaros) que las variedades y ecotipos de color, situación que no fue evidenciada en nuestro trabajo.

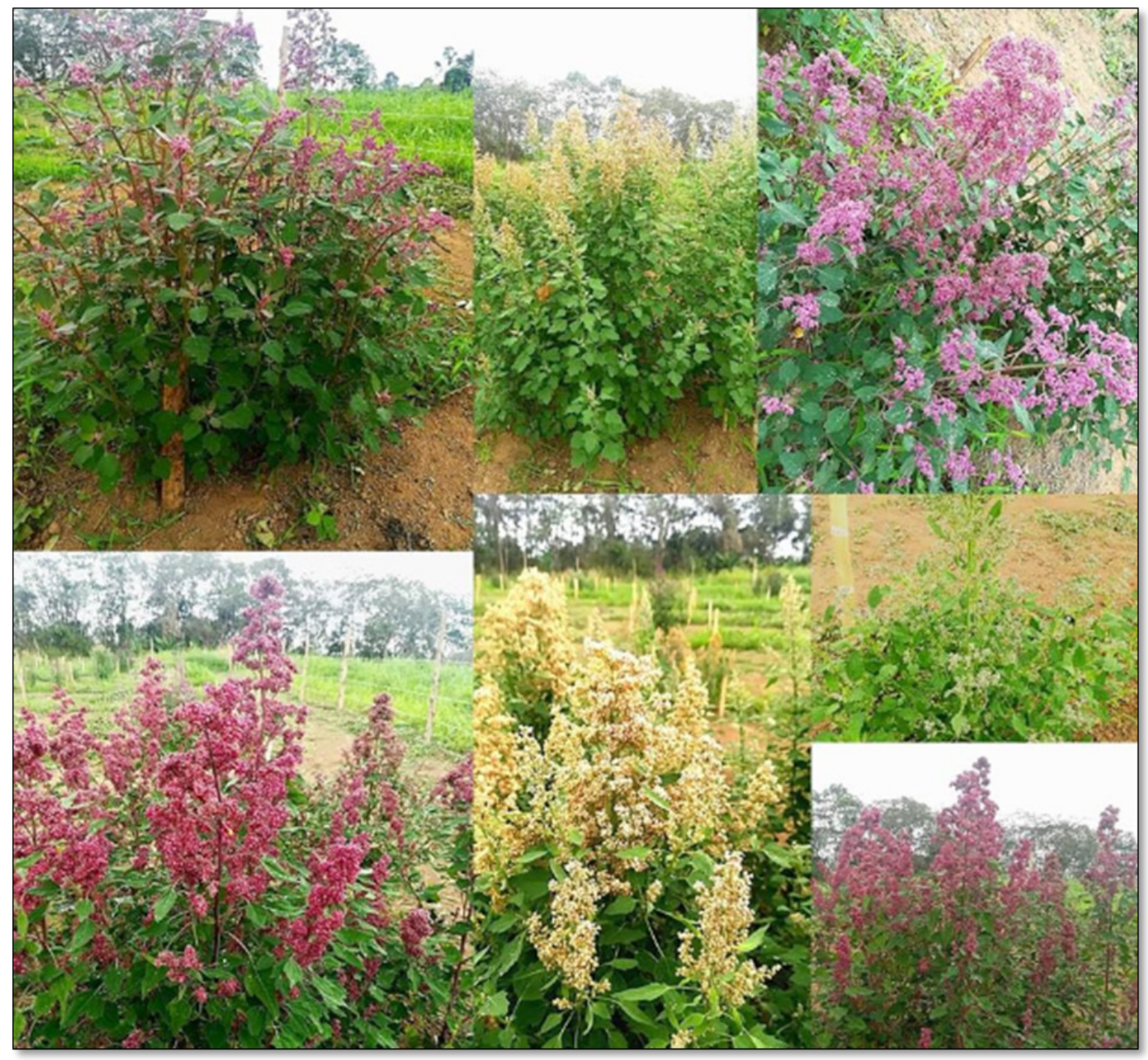

Figura 1. Variabilidad fenotípica de los genotipos de quinua con las condiciones agroecológicas de la Finca Experimental "La María". Genotipos rojos, blancos y amarillos. 
Cuadro 2. Variables cualitativas de las colecciones de quinua evaluadas en la Finca Experimental "La María".

\begin{tabular}{|c|c|c|c|c|c|c|c|c|c|c|c|c|c|c|c|}
\hline \multicolumn{4}{|c|}{ Tipo de panoja } & \multicolumn{4}{|c|}{ Color de hoja } & \multicolumn{4}{|c|}{ Color de flor } & \multicolumn{4}{|c|}{ Color del grano } \\
\hline Genotipo & Glo. & Ama. & Mix. & $\begin{array}{l}\text { Ama } \\
\text { - Ver. }\end{array}$ & Ros. & Ama. & Nar. & Ver. & Ama. & Roj. & $\begin{array}{l}\text { Ros - } \\
\text { Ama. }\end{array}$ & Ama. & BI. & Roj. & Neg. \\
\hline 26 & $\mathrm{X}$ & & & $\mathrm{X}$ & & & & & & & $\mathrm{X}$ & & & $\mathrm{X}$ & \\
\hline 36 & $\mathrm{X}$ & & & $\mathrm{X}$ & & & & $\mathrm{X}$ & & & & & & & $\mathrm{X}$ \\
\hline 41 & & $\mathrm{X}$ & & & $\mathrm{X}$ & & & & $\mathrm{X}$ & & & $\mathrm{X}$ & & & \\
\hline 42 & & $\mathrm{X}$ & & $\mathrm{X}$ & & & & & $\mathrm{X}$ & & & & $\mathrm{X}$ & & \\
\hline 48 & $\mathrm{X}$ & & & & & & $\mathrm{X}$ & $\mathrm{X}$ & & & & $\mathrm{X}$ & & & \\
\hline 49 & & $\mathrm{X}$ & & & $\mathrm{X}$ & & & $\mathrm{X}$ & & & & $\mathrm{X}$ & & & \\
\hline 52 & $\mathrm{X}$ & & & & & & $\mathrm{X}$ & $\mathrm{X}$ & & & & $\mathrm{X}$ & & & \\
\hline 54 & $\mathrm{X}$ & & & $\mathrm{X}$ & & & & $\mathrm{X}$ & & & & $\mathrm{X}$ & & & \\
\hline $\mathrm{O}-1$ & $\mathrm{X}$ & & & & & $\mathrm{X}$ & & $\mathrm{X}$ & & & & & $\mathrm{X}$ & & \\
\hline $\mathrm{O}-2$ & $\mathrm{X}$ & & & $\mathrm{X}$ & & & & $\mathrm{X}$ & & & & & $\mathrm{X}$ & & \\
\hline $\mathrm{O}-3$ & $\mathrm{X}$ & & & $\mathrm{X}$ & & & & & & $\mathrm{X}$ & & & & $\mathrm{X}$ & \\
\hline $\begin{array}{l}\mathrm{O}-4 \\
\mathrm{O}-5\end{array}$ & $\mathrm{x}$ & $\mathrm{X}$ & & $\mathrm{X}$ & $\mathrm{X}$ & & & & $\begin{array}{l}X \\
X\end{array}$ & & & $\begin{array}{l}X \\
X\end{array}$ & & & \\
\hline$O-6$ & & & $\mathrm{X}$ & & $\mathrm{X}$ & & & & & $\mathrm{X}$ & & & & $\mathrm{X}$ & \\
\hline $\mathrm{O}-7$ & & $\mathrm{X}$ & & & & $\mathrm{X}$ & & & & $\mathrm{X}$ & & & & $\mathrm{X}$ & \\
\hline $\mathrm{O}-8$ & $\mathrm{X}$ & & & & & $\mathrm{X}$ & & $\mathrm{X}$ & & & & & $\mathrm{X}$ & & \\
\hline$O-9$ & $\mathrm{X}$ & & & & & $\mathrm{X}$ & & $\mathrm{X}$ & & & & & $\mathrm{X}$ & & \\
\hline$O-10$ & & $\mathrm{X}$ & & & $\mathrm{X}$ & & & & & & $\mathrm{X}$ & $\mathrm{X}$ & & & \\
\hline RGG & $\mathrm{X}$ & & & $\mathrm{X}$ & & & & $\mathrm{X}$ & & & & & $\mathrm{X}$ & & \\
\hline $\mathrm{J} 4$ - O10 & $\mathrm{X}$ & & & $\mathrm{X}$ & & & & $\mathrm{X}$ & & & & & & $\mathrm{X}$ & \\
\hline Faro 2 & & & $\mathrm{X}$ & & & & $\mathrm{X}$ & $\mathrm{X}$ & & & & $\mathrm{X}$ & & & \\
\hline Número & 13 & 6 & 2 & 9 & 5 & 4 & 3 & 12 & 4 & 3 & 2 & 9 & 6 & 5 & 1 \\
\hline Porcentaje & 62 & 29 & 9 & 43 & 24 & 19 & 14 & 57 & 19 & 14 & 10 & 43 & 28 & 24 & 5 \\
\hline
\end{tabular}

Glo $=$ Glomerulada, Ama $=$ Amarantiforme, Mix $=$ Mixtas, Ama - Ver $=$ Amarillo - verdoso, Ros $=$ Rosado, Ama=Amarillo, Nar=Naranja, Ver=Verde, Ama $=$ Amarillo, Roj=Roja, Ros - Ama $=$ Rosa - amarillo, $\mathrm{Ama}=$ Amarillo; $\mathrm{Bl}=$ Blanco, $\mathrm{Roj}=:$ Rojo, Neg= Negro. 


\section{Altura de planta (cm)}

En el Cuadro 3 se detallan los resultados obtenidos en el estudio de la variable altura. En el análisis de varianza se encontró una alta significancia estadística a los 30, 60 y 90 días. La prueba de Tukey $(\mathrm{p}<0.05)$, mostró que el genotipo que alcanzó la mayor altura fue el 48 con $154.17 \mathrm{~cm}$ a los 90 días (Cuadro 3). Registrar la mayor altura en uno de los genotipos chilenos, ratifica lo manifestado por Gómez y Aguilar (2016), quien señala que quinuas del nivel del mar o costeras son plantas más o menos vigorosas, de 100 a $140 \mathrm{~cm}$ de altura. Además cabe resaltar que al ser la altura un rasgo con variabilidad cuantitativa (Cuahutémoc et al., 2013) es muy dependiente del ambiente y de la interacción que el genotipo alcance con el mismo. Por ejemplo, Apaza (2006) encontró alturas con promedios de plantas de $103.73 \mathrm{~cm}$ al evaluar el comportamiento agronómico de diez variedades de quinua en las comunidades del Ayllu Huatari y de la zona intersalar del (Uyuni - Coipasa) Bolivia.

\section{Diámetro del tallo $(\mathrm{cm})$}

De acuerdo al análisis de varianza los genotipos de quinua no presentaron significancia estadística en la variable diámetro del tallo a los 30 días, mientras que en los 60 y 90 días si presentaron alta significancia. Según Tukey $(p<0.05)$ el genotipo que alcanzó el mayor diámetro fue el $\mathrm{O}-2 \mathrm{con} 1.54 \mathrm{~cm}$ a $\operatorname{los} 90$ días (Cuadro 4). De acuerdo al estudio realizado por Luzón (2016) el análisis de varianza para el diámetro del tallo, muestra diferencias estadísticas, siendo la media entre tratamientos de 2.52 .

\section{Días a la cosecha}

Los genotipos que presentaron madurez fisiológica en menor tiempo oscilaron entre los 90 días y 107 días a la cosecha. En los genotipos que se prolongó la madurez fisiológica contaron con 127 a 143 días a la cosecha (Cuadro 5). Al respecto Tapia (2014), afirma la existencia de razas en amiláceas y vitrias, clasificándolas en tres periodos vegetativos: precoces los que van de 130 a 150 días; intermedios, de 150 a 180 días; y tardíos de más de 180 días. A su vez Delgado et al. (2009), encontró precocidad en genotipos de quinua dulce evaluados en el departamento de Nariño - Colombia con días a madurez fisiológica entre 129 a 135 días. La amplitud de rangos de los ciclos de cultivo en las plantas está determinado por el tiempo térmico, también conocido como metodología de grados día $\left({ }^{\circ} \mathrm{Cd}\right)$ o acumulación de unidades de calor; que con base en temperaturas máximas y mínimas diarias da un índice del calor utilizable diario, cuyo acumulado de valores permite el desarrollo de sucesivas etapas fisiológicas del cultivo (Trudgill et al., 2005; Parthasarathi et al., 2013). Es por esta razón que los cultivos sembrados en esta zona del Ecuador, con temperaturas diarias promedio de $24{ }^{\circ} \mathrm{C}$ alcanzaron mayoritariamente ciclos precoces, insertando al cultivo de quinua como una nueva alternativa en la rotación de los cultivos de ciclo corto.

\section{Peso de mil semillas}

Los genotipos que obtuvieron los pesos más relevantes fueron: Faro 2, 52, 41 y 36 con pesos de $2.58 \mathrm{~g}, 2.53 \mathrm{~g}, 2.49 \mathrm{~g}$ y también $2.49 \mathrm{~g}$ respectivamente (Cuadro 6). Al respecto, Choque (2010) obtuvo peso de mil semillas de $4.49 \mathrm{~g}$, la cual pertenece a la variedad Real blanca, atribuible al gran tamaño de granos 2.5 mm. Haber obtenido pesos bajos está relacionado con el criterio de que, al haberse acortado el ciclo productivo de la quinua en esta zona, los periodos de desarrollo y llenado de grano también se vieron acortados impidiendo que los granos puedan crecer y acumular mayores reservas (Lesjak y Calderini, 2017).

\section{Rendimiento de grano por planta (g)}

Los mejores rendimientos por planta de quinua fueron alcanzados por los genotipos $\mathrm{O}-5$ con $143.15 \mathrm{~g}, \mathrm{O}-2$ con $95.87 \mathrm{~g}, \mathrm{O}-8$ con $83.60 \mathrm{~g}$ y por último el genotipo 48 con $81.78 \mathrm{~g}$, a diferencia del primer genotipo, los cuatro genotipos mencionados anteriormente son estadísticamente iguales entre sí (Cuadro 7). Según Tukey $(\mathrm{p}<0.05)$, si se encontraron diferencias estadísticas entre las medias. En cambio, Veloza et al., (2016), en la zona andina de Colombia obtuvo rendimientos variados entre 140.09 a 260.74 g/planta; ciertos genotipos de quinua, denominadas Piartal, Nariño y Bolivia, alcanzaron el desarrollo fenológico más precoz a los seis meses. Los principales componentes del rendimiento son el peso de las semillas y el número de semillas (Gambín \&y Borrás, 2010). Como se ha manifestado anteriormente el efecto de la temperatura generó un bajo peso de los granos, pero en la mayoría de los casos esto no reflejó un bajo rendimiento, lo que podría estar dado por un mayor número de granos por unidad de superficie. Además, los rendimientos de un genotipo están dados por la interacción genotipo $\mathrm{x}$ ambiente, tal como se puede observar en el genotipo RGG, originario del sur de Chile, quien logró $6.36 \mathrm{~g} /$ planta, lo que equivale a 84.8 $\mathrm{g} \mathrm{m}^{2}$, rendimientos sumamente bajos en comparación con los obtenidos por Lesjak y Calderini (2017) en el sur de Chile con $524.4 \mathrm{~g} \mathrm{~m}^{2}$. 


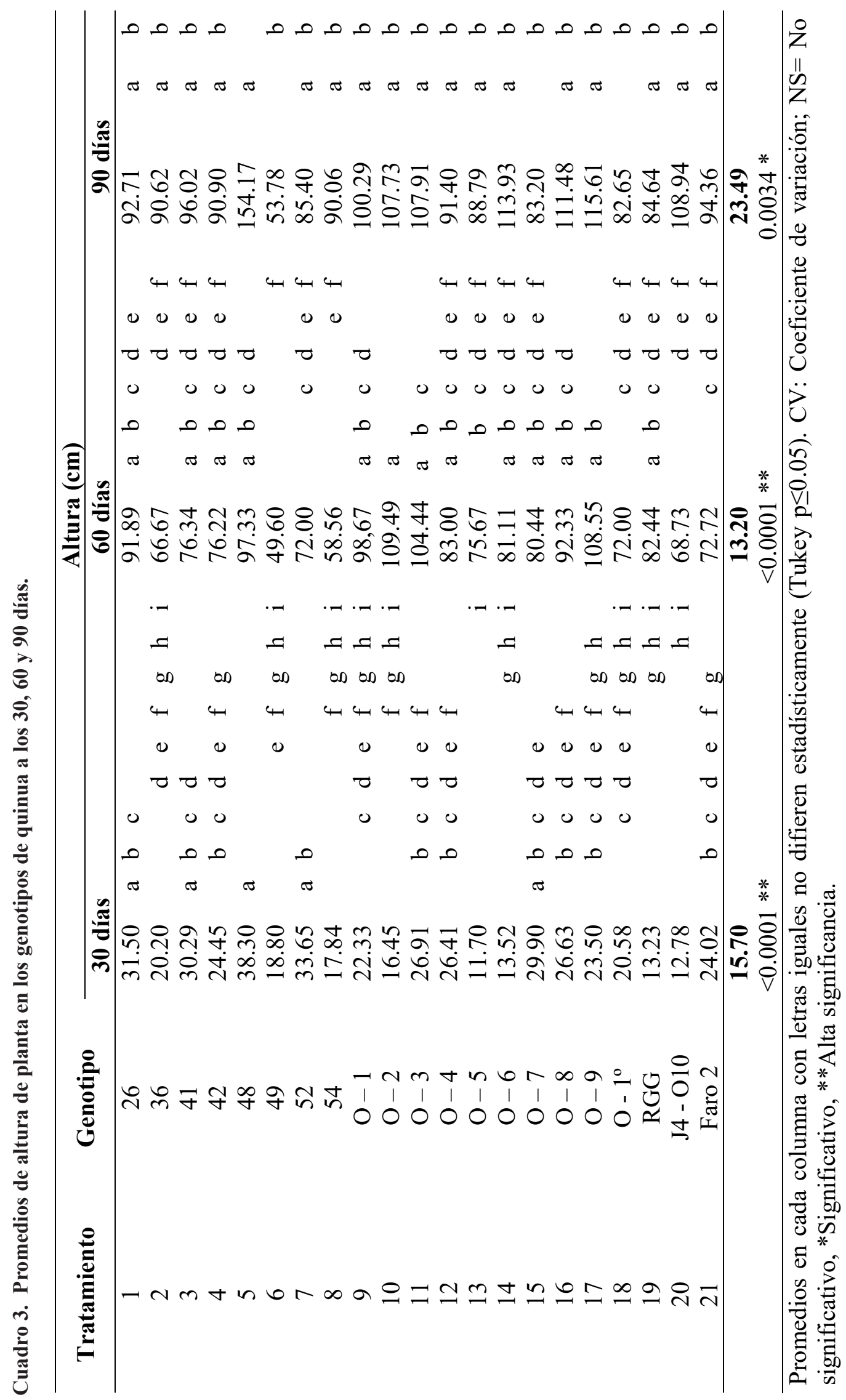


Cuadro 4. Promedios de diámetro del tallo en los genotipos de quinua a los 30, 60 y 90 días.

\begin{tabular}{|c|c|c|c|c|c|c|c|c|c|c|c|c|c|}
\hline \multirow{3}{*}{$\begin{array}{c}\begin{array}{c}\text { Trata- } \\
\text { miento }\end{array} \\
1\end{array}$} & \multirow{3}{*}{$\begin{array}{c}\begin{array}{c}\text { Genoti- } \\
\text { po }\end{array} \\
26\end{array}$} & \multicolumn{12}{|c|}{ Diámetro (cm) } \\
\hline & & \multicolumn{2}{|c|}{30 días } & \multicolumn{6}{|c|}{60 días } & \multicolumn{4}{|c|}{90 días } \\
\hline & & 0.44 & $\mathrm{a}$ & 1.38 & $\mathrm{a}$ & $\mathrm{b}$ & & & & 1.49 & $\mathrm{a}$ & & \\
\hline 2 & 36 & 0.27 & $\mathrm{a}$ & 0.96 & $\mathrm{a}$ & $\mathrm{b}$ & $\mathrm{c}$ & d & $\mathrm{e}$ & 1.12 & $\mathrm{a}$ & $\mathrm{b}$ & $\mathrm{c}$ \\
\hline 3 & 41 & 0.37 & $\mathrm{a}$ & 0.61 & & & & d & $\mathrm{e}$ & 0.72 & & $\mathrm{~b}$ & $\mathrm{c}$ \\
\hline 4 & 42 & 0.25 & $\mathrm{a}$ & 0.87 & $\mathrm{a}$ & $\mathrm{b}$ & $\mathrm{c}$ & d & $\mathrm{e}$ & 0.90 & $\mathrm{a}$ & $\mathrm{b}$ & $\mathrm{c}$ \\
\hline 5 & 48 & 0.44 & $\mathrm{a}$ & 1.38 & $\mathrm{a}$ & $\mathrm{b}$ & & & & 1.49 & $\mathrm{a}$ & & \\
\hline 6 & 49 & 0.25 & $\mathrm{a}$ & 0.68 & & & $\mathrm{c}$ & d & $\mathrm{e}$ & 0.83 & $\mathrm{a}$ & $\mathrm{b}$ & $\mathrm{c}$ \\
\hline 7 & 52 & 0.39 & $\mathrm{a}$ & 0.75 & & & $\mathrm{c}$ & d & $\mathrm{e}$ & 0.69 & & $\mathrm{~b}$ & $\mathrm{c}$ \\
\hline 8 & 54 & 0.22 & $\mathrm{a}$ & 0.60 & & & & $d$ & $\mathrm{e}$ & 0.93 & $\mathrm{a}$ & b & $\mathrm{c}$ \\
\hline 9 & O - 1 & 0.36 & $\mathrm{a}$ & 1.11 & $\mathrm{a}$ & $\mathrm{b}$ & $\mathrm{c}$ & $d$ & $\mathrm{e}$ & 1.14 & $\mathrm{a}$ & $\mathrm{b}$ & $\mathrm{c}$ \\
\hline 10 & $\mathrm{O}-2$ & 0.28 & $\mathrm{a}$ & 1.47 & $\mathrm{a}$ & & & & & 1.54 & $\mathrm{a}$ & & \\
\hline 11 & $\mathrm{O}-3$ & 0.42 & $\mathrm{a}$ & 1.27 & $\mathrm{a}$ & $\mathrm{b}$ & $\mathrm{c}$ & & & 1,18 & $\mathrm{a}$ & $\mathrm{b}$ & $\mathrm{c}$ \\
\hline 12 & $\mathrm{O}-4$ & 0.35 & $\mathrm{a}$ & 0.82 & & $\mathrm{~b}$ & $\mathrm{c}$ & d & $\mathrm{e}$ & 0.85 & $\mathrm{a}$ & b & $\mathrm{c}$ \\
\hline 13 & O - 5 & 0.27 & $\mathrm{a}$ & 1.17 & $\mathrm{a}$ & $\mathrm{b}$ & $\mathrm{c}$ & d & & 1.30 & $\mathrm{a}$ & b & \\
\hline 14 & O - 6 & 0.26 & $\mathrm{a}$ & 0.73 & & & $\mathrm{c}$ & d & e & 0.97 & $\mathrm{a}$ & b & $\mathrm{c}$ \\
\hline 15 & O - 7 & 0.40 & $\mathrm{a}$ & 0.71 & & & $\mathrm{c}$ & $d$ & $\mathrm{e}$ & 0.69 & & b & $\mathrm{c}$ \\
\hline 16 & O - 8 & 0.37 & $\mathrm{a}$ & 1.14 & $\mathrm{a}$ & $\mathrm{b}$ & $\mathrm{c}$ & d & & 1.31 & $\mathrm{a}$ & b & \\
\hline 17 & O - 9 & 0.33 & $\mathrm{a}$ & 1.07 & $\mathrm{a}$ & $\mathrm{b}$ & $\mathrm{c}$ & $\mathrm{d}$ & $\mathrm{e}$ & 1.23 & $\mathrm{a}$ & b & $\mathrm{c}$ \\
\hline 18 & $O-10$ & 0.22 & $\mathrm{a}$ & 0.64 & & & & $\mathrm{~d}$ & $\mathrm{e}$ & 0.53 & & & $\mathrm{c}$ \\
\hline 19 & RGG & 0.27 & $\mathrm{a}$ & 0.82 & & $\mathrm{~b}$ & $\mathrm{c}$ & $\mathrm{d}$ & $\mathrm{e}$ & 1.00 & $\mathrm{a}$ & b & $\mathrm{c}$ \\
\hline 20 & $\mathrm{~J} 4$ - O10 & 0.25 & $\mathrm{a}$ & 0.51 & & & & & $\mathrm{e}$ & 0.78 & & b & $\mathrm{c}$ \\
\hline 21 & Faro 2 & 0.72 & $\mathrm{a}$ & 0.75 & & & $\mathrm{c}$ & $\mathrm{d}$ & $\mathrm{e}$ & 0.94 & $\mathrm{a}$ & b & $\mathrm{c}$ \\
\hline C.V $(\%)$ & & 50.54 & & 21.39 & & & & & & 22.60 & & & \\
\hline$(\mathrm{p}<0.05)$ & & 0.216 & & $<0.000$ & & & & & & $<0.00$ & $* *$ & & \\
\hline
\end{tabular}

Promedios en cada columna con letras iguales no difieren estadísticamente (Tukey $\mathrm{p} \leq 0.05$ ). CV: Coeficiente de variación; NS= no significativo; *Significativo, **Alta significancia. 

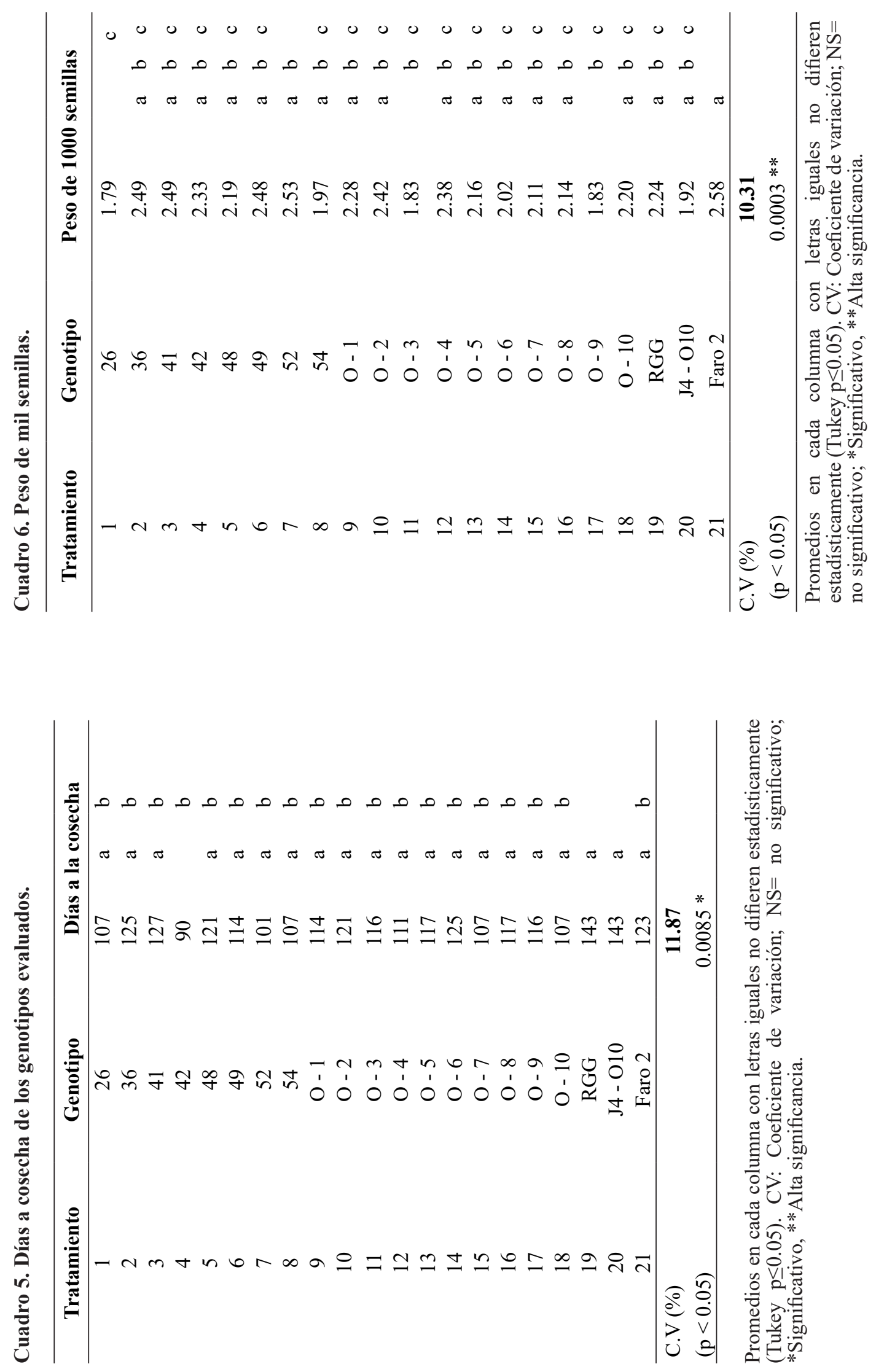
Cuadro 7. Rendimiento de grano por planta de los genotipos de quinua (g).

\begin{tabular}{|c|c|c|c|c|c|c|c|c|c|c|}
\hline Tratamiento & Genotipo & & end & aien & pc & pla & ta $(g$ & & & \\
\hline 1 & 26 & 33.96 & & & & $\mathrm{~d}$ & $\mathrm{e}$ & $\mathrm{f}$ & $\mathrm{g}$ & \\
\hline 2 & 36 & 32.84 & & & & $\mathrm{~d}$ & $\mathrm{e}$ & $\mathrm{f}$ & $\mathrm{g}$ & $\mathrm{h}$ \\
\hline 3 & 41 & 12.30 & & & & & & $\mathrm{f}$ & g & $\mathrm{h}$ \\
\hline 4 & 42 & 15.97 & & & & & $\mathrm{e}$ & $\mathrm{f}$ & $\mathrm{g}$ & $\mathrm{h}$ \\
\hline 5 & 48 & 81.78 & & $\mathrm{~b}$ & & & & & & \\
\hline 6 & 49 & 39.51 & & & & $\mathrm{~d}$ & $\mathrm{e}$ & $\mathrm{f}$ & & \\
\hline 7 & 52 & 4.87 & & & & & & & & $\mathrm{~h}$ \\
\hline 8 & 54 & 12.97 & & & & & & $\mathrm{f}$ & g & $\mathrm{h}$ \\
\hline 9 & $\mathrm{O}-1$ & $4 ., 61$ & & & $\mathrm{c}$ & $\mathrm{d}$ & & & & \\
\hline 10 & $\mathrm{O}-2$ & 95.87 & & $\mathrm{~b}$ & & & & & & \\
\hline 11 & $\mathrm{O}-3$ & 35.49 & & & & $\mathrm{~d}$ & $\mathrm{e}$ & $\mathrm{f}$ & & \\
\hline 12 & $\mathrm{O}-4$ & 41.96 & & & $\mathrm{c}$ & $\mathrm{d}$ & $\mathrm{e}$ & & & \\
\hline 13 & $\mathrm{O}-5$ & 143.15 & $\mathrm{a}$ & & & & & & & \\
\hline 14 & $O-6$ & 18.32 & & & & & $\mathrm{e}$ & $\mathrm{f}$ & g & $\mathrm{h}$ \\
\hline 15 & $\mathrm{O}-7$ & 21.98 & & & & $\mathrm{~d}$ & $\mathrm{e}$ & $\mathrm{f}$ & $\mathrm{g}$ & $\mathrm{h}$ \\
\hline 16 & $\mathrm{O}-8$ & 83.60 & & $\mathrm{~b}$ & & & & & & \\
\hline 17 & $O-9$ & 68.87 & & $\mathrm{~b}$ & $\mathrm{c}$ & & & & & \\
\hline 18 & $\mathrm{O}-1^{\mathrm{o}}$ & 27.95 & & & & $\mathrm{~d}$ & $\mathrm{e}$ & $\mathrm{f}$ & $\mathrm{g}$ & $\mathrm{h}$ \\
\hline 19 & RGG & 6.36 & & & & & & & $\mathrm{~g}$ & $\mathrm{~h}$ \\
\hline 20 & $\mathrm{~J} 4$ - O10 & 12.17 & & & & & & $\mathrm{f}$ & $\mathrm{g}$ & $\mathrm{h}$ \\
\hline 21 & Faro 2 & 16.74 & & & & & $\mathrm{e}$ & $\mathrm{f}$ & $\mathrm{g}$ & $\mathrm{h}$ \\
\hline C.V (\%) & & 22.19 & & & & & & & & \\
\hline$(\mathrm{p}<0.05)$ & & $<0.0001 * *$ & & & & & & & & \\
\hline
\end{tabular}

Promedios en cada columna con letras iguales no difieren estadísticamente (Tukey $\mathrm{p} \leq 0.05$ ). CV: Coeficiente de variación; NS= no significativo; *Significativo, **Alta significancia.

\section{Conclusiones}

$\mathrm{L}$ os materiales más sobresalientes en cuanto a precocidad, altura y diámetro de la planta fueron: los genotipos 48, $\mathrm{O}-9$ y el genotipo que destacó en productividad es el $\mathrm{O}-5$. Estos registros demuestran resultados positivos en cuanto a la adaptación de genotipos de quinua provenientes de zonas costeras de otros países a las condiciones agroclimáticas en la zona explorada, dado que los materiales ecuatorianos son estrictamente de tipo andino. Restaría realizar estudios en distintas épocas y localidades para respaldar lo evaluado en la presente investigación.

\section{Financiamiento}

Este proyecto fue financiado con Fondos Concursables FOCICYT-2017 Quinta Convocatoria, de la Universidad Técnica Estatal de Quevedo.

\section{Bibliografía}

Alvarado, M.C. \& Martínez, A.G. (2015). Estudio de Factibilidad Para La Producción de Quinua En Las Comunidades Del Cantón Colta, Provincia de Chimborazo y Propuesta de Plan de Exportación Al Mercado Francés. Universidad Politécnica Salesiana.

Bazile, D., Bertero, D., Nieto, C. (2013). Estado Del Arte Del La Quinua En El Mundo En 2013.

Belmonte, C., Soares de Vasconcelos, E., Tsutsumi, C.Y., Lorenzeti, E., Hendges, C., Coppo, J.C., da Silva Martinez, A., Pan, R., Santos Brito, T. \& Inagaki, A.M. (2018). Agronomic and Productivity Performance for Quinoa Genotypes in an Agroecological and Conventional Production System. American Journal of Plant Sciences, 09, 880-891.

Cortés, A.X. \& Rubiano, A.J. (2007). Caracterización de tres ecotipos de Quinua "Chenopodium quinoa Willd" Mediante Técnicas Agroecológicas, en dos zonas agroclimatologicamente diferentes del Departamento de Cundinamarca. Inventum, 2, 13. 
Cuahutémoc, V., Hernández, R., De La, M., Olán, O., Rangel, E.E., SANGerman-Jarquín, D.M., Hernández Casillas, J.M. \& Schwentesius De Rindermann, R. (2013). Variabilidad cualitativa y cuantitativa de accesiones de amaranto determinada mediante caracterización morfológica* Qualitative and quantitative variability determined through morphological characterization in amaranth accessions. Revista Mexicana de Ciencias Agrícolas, 4, 789-801.

CRUCES, L. (2016). Quinua manejo integrado de plagas (1 ed.). (T. Santivañez, \& B. Jara, Edits.) Santiago, Chile: NEC Proyecto Sierra.

Delgado P., A.I., Palacios C., J.H. \& Betancourt G., C. (2009). Evaluación de 16 genotipos de quinua dulce (Chenopodium quinoa willd) en el municipio de Iles, Nariño (Colombia). Agronomía Colombiana, 27, 159167.

FAOSTAT. (2017). Food and Agriculture Organization of the United Nations Statistical Databases. FAO-FAOSTAT, http://faostat.fao.org/site/567/DesktopDefault. aspx?PageID=567\#ancor

Fuentes, F., Martinez, E., Hinrichsen, P., Jellen, E. \& Maughan, P. (2009). Assessment of genetic diversity patterns in Chilean quinoa (Chenopodium quinoa Willd.) germplasm using multiplex fluorescent microsatellite markers. Conservation Genetics, 10, 369-377.

GAMBín, B.L. \& Borrás, L. (2010). Resource distribution and the trade-off between seed number and seed weight: A comparison across crop species. Annals of Applied Biology, 156, 91-102.

Gómez, L. \& Aguilar, E. (2016). Guía de Cultivo de La Quinua, Universida. Lima - Perú.

HERNÁNDEZ, J. (2015). La quinua, una opción para la nutrición del paciente con diabetes mellitus. Revista Cubana de Endocrinología, 26, 304-312.

INIA. (2015). Instituto de Investigaciones Agropecuarias. Quínoa: Un súper alimento para Chile y el mundo. Tierra Adentro, 108, 84.

LesJak, J. \& CALDERINI, D.F. (2017). Increased Night Temperature Negatively Affects Grain Yield, Biomass and Grain Number in Chilean Quinoa. Frontiers in Plant Science, 8, 1-11.

Long, N.V. (2016). Effects of salinity stress on growth and yield of quinoa (Chenopodium quinoa Willd.) at flower initiation stages. Vietnam J. Agric. Sci., 14, 321-327.

Lutz, M., Martínez, A. \& Martínez, E. (2013). Daidzein and Genistein contents in seeds of quinoa (Chenopodium quinoa Willd.) from local ecotypes grown in arid Chile. Industrial Crops and Products, 49, 117-121.

MujicA, A. \& JACOBSEN, S.-E. (2006). La quinua (Chenopodium quinoa Willd.) y sus parientes silvestres. Botánica Económica de los Andes Centrales, 449-457.

Parthasarathi, T., Velu, G. \& Jeyakumar, P. (2013). Impact of Crop Heat Units on Growth and Developmental
Physiology of Future Crop Production: A Review. Research \& Reviews: A Journal of Crop Science and Technology, 2, 2319-3395.

Rojas, W., Soto, J., Pinto, M. \& Jager, M. (2010). Granos Andinos.

Thanapornpoonpong, S.N., Vearasilp, S., Pawelzik, E. $\&$ Gorinstein, S. (2008). Influence of various nitrogen applications on protein and amino acid profiles of amaranth and quinoa. Journal of Agricultural and Food Chemistry, 56, 11464-11470.

Trudgill, D.L., HoneK, A., Li, D. \& Van Straalen, N.M. (2005). Thermal time - Concepts and utility. Annals of Applied Biology, 146, 1-14.

Zurita-Silva, A., Fuentes, F., Zamora, P., Jacobsen, S.E. \& Schwember, A.R. (2014). Breeding quinoa (Chenopodium quinoa Willd.): potential and perspectives. Molecular Breeding, 34, 13-30. 